\title{
Event-triggered state-feedback control for continuous-time plants subject to input saturation
}

\author{
L. G. Moreira • L. B. Groff • J. M. Gomes da Silva Jr.
}

Received: date / Accepted: date

\begin{abstract}
This paper proposes a method to design event-triggered state-feedback controllers for continuous-time LTI plants subject to input saturation. Using Lyapunov theory techniques, LMI-based conditions are derived to guarantee regional (or global, when possible) asymptotic stability of the origin. Optimization problems are proposed as means to tune the trigger parameters aiming at reducing the sampling activity. Co-design of the trigger function and of the control law is also addressed, as well as the extension of the results to a class of uncertain systems. Simulations illustrate the application of the methods.
\end{abstract}

Keywords Event-trigger - Saturation · Networked Control Systems · Linear Plants

\section{Introduction}

Nowadays many control systems are implemented over general purpose shared networks to take advantage of the effectiveness in terms of cost and flexibility that such networks offer. In this configuration, the communication between sensors, controllers and actuators is done using the network and occurs at discrete time instants. This scenario describes what is commonly known as networked control systems (NCS) (???). This field has attracted attention since the 1990's decade, as shown in ? and the references cited therein. Issues such as communication bandwidth and energy consumption

L. G. Moreira · L. B. Groff - J. M. Gomes da Silva Jr.

Department of Automation and Energy

Universidade Federal do Rio Grande do Sul

Av. Osvaldo Aranha 103, 90035-190 Porto Alegre-RS, Brazil.

Tel.: +55 51-3308-3140

Fax: +55 51-3308-3293

E-mail: jmgomes@ece.ufrgs.br arise from this distributed paradigm (??) and have been addressed with different techniques, among them, event-triggered control.

In an event-triggered control system, data is sampled and transmitted only when a trigger condition is violated. In this context, it is of primary importance to develop systematic methods to tune the trigger aiming at reducing the number of data transmissions while ensuring the stability of the closed-loop system. In ?, a threshold on the relative state measurement error is used in the trigger condition and it is shown that the resulting inter-event times are lower-bounded. More recently, other issues have been addressed in the literature: the problem of designing an event-trigger strategy for state-feedback controlled single-input-single-output linear plants using a state observer is addressed in ? for continuous-time systems and in ? for discrete-time systems. The co-design between the trigger function and controller gains for LTI plants is addressed in ?. In ?, a design method for event-triggered PI controllers that uses a quadratic criterion for the triggering function and for the tuning of the controller parameters is presented.

On the other hand, control input saturation is a phenomenon present in almost all control systems (?). In the context of event-triggered control, algorithms based on linear-quadratic criteria to design periodic, event-triggered and self-triggered controllers are proposed in ?, taking explicitly into account the actuator saturation. In ?, an event-triggered controller is designed without considering the saturation and examples of how the saturation impacts the performance of the system are given. In ?, stability issues under control saturation are addressed considering PI and generic dynamic output feedback controllers. For discrete-time systems, ? addresses saturating state-feedback control laws. 
The present paper addresses the design of an eventtriggered state-feedback strategy for continuous-time linear plants subject to input saturation, considering that the plant and the controller are in different nodes and that the control signal is kept constant between two successive sampling instants. Differently from ?, instead of using hybrid systems theory, a direct Lyapunovbased approach is applied to derive the stability conditions. In contrast to ?, which focus on estimating the region of attraction of the origin for a given control law and given trigger parameters, we address the problem of designing these parameters in order to ensure that a given region of interest is included in the region of attraction of the closed-loop system under the eventtrigger strategy. Moreover, the co-design of the trigger parameters and of the control law is also addressed. The trigger function considered in this paper takes into account a weighted deviation between the last sampled state and the current one. Stability conditions in the form of linear matrix inequalities (LMIs) are derived and since the parameters of the trigger function appear explicitly in these conditions, convex optimization problems aiming at reducing the sampling activity are proposed as means to select these parameters. Numerical examples illustrate the application of the methods.

The paper is organized as follows: Section 2 presents basic definitions and formally states the problem we address. Section 3 recalls stability conditions for continuous-time (non-triggered) LTI systems subject to input saturation. Section 4 presents our event-trigger strategy and the stability conditions for the closed-loop system in regional and global contexts, considering that the controller gains were previously designed. Convex optimizations problems suitable for selecting the trigger parameters aiming at reducing the sampling activity are presented in Section 4.1. Section 5 extends the results from previous section showing how to design the trigger parameters and the controller gains simultaneously (co-design). Section 6 illustrates the application of the method.

\subsection{Notation}

- $\mathbb{R}$ represents the set of real numbers.

- The saturation function is defined as

$$
\operatorname{sat}(v) \triangleq \begin{cases}-u_{0} & \text { if } v<-u_{0} \\ v & \text { if }-u_{0} \leq v \leq u_{0} \\ u_{0} & \text { if } v>u_{0}\end{cases}
$$

if $v$ is a vector, the definition holds element-wise.

- $A^{\prime}$ denotes the transpose of matrix $A$.

- $\operatorname{He}\{A\} \triangleq A+A^{\prime}$.

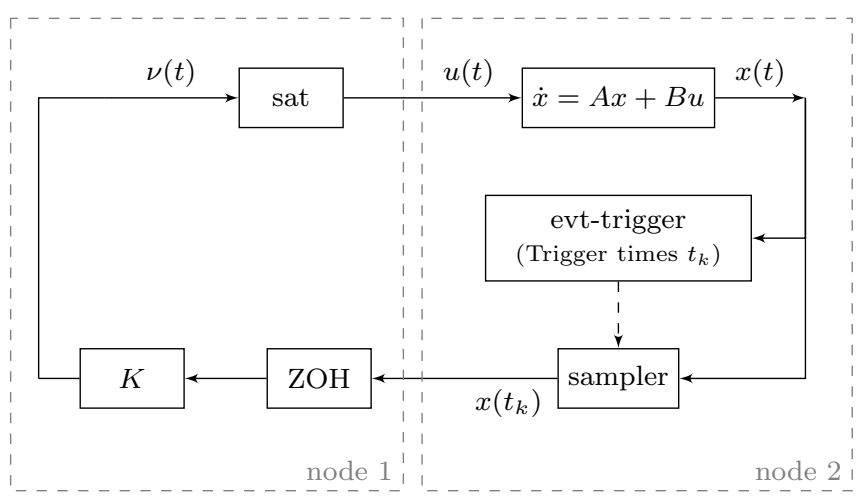

Fig. 1 Closed-loop system diagram

- The symbol $*$ stands for symmetric blocks within a matrix.

- $\operatorname{tr}(A)$ denotes the trace of matrix $A$.

- $A_{(j)}$ and $x_{(j)}$ denote the j-th row of matrix $A$ and vector $x$, respectively.

- $\operatorname{diag}(X, Y)$ denotes the block-diagonal matrix composed by the blocks $X$ and $Y$.

- $x \preceq y$ with $x, y \in \mathbb{R}^{n}$ means that $x_{(j)} \leq y_{(j)}, \forall j=$ $1 \ldots n$.

- $\mathcal{E}(P)=\left\{x \in \mathbb{R}^{q} ; x^{\prime} P x \leq 1\right\}$ is the ellipsoid defined by a matrix $P \in \mathbb{R}^{q \times q}, P=P^{\prime}>0$.

- $\|\cdot\|$ denotes the Euclidean norm.

- $\mathrm{Co}\{\cdot\}$ denotes a convex hull.

\section{Problem Statement}

Consider a continuous-time linear plant defined by the following equation:

$\dot{x}(t)=A x(t)+B u(t)$

where $x \in \mathbb{R}^{n}$ is the state vector; $u \in \mathbb{R}^{m}$ is the input, limited in amplitude such that $-u_{0} \preceq u \preceq u_{0}$, with $u_{0} \succ 0 \in \mathbb{R}^{m} ; A$ and $B$ are real-valued constant matrices of appropriate dimensions. We assume that the plant is observable and controllable.

We consider a networked control implementation where plant and controller are in separate nodes and are connected through a general purpose network forming the closed-loop system depicted in Figure 1.

At instants $t_{k}, k=0,1,2, \ldots$ determined by the event-trigger generator, a sample of the plant state is sent to the controller node. Between two trigger instants, the controller input is held at the constant sampled value by means of zero-order holders. We assume $t_{0}=0$.

Therefore, the closed-loop system reads:

$\left\{\begin{array}{l}\dot{x}(t)=A x(t)+B u(t) \\ u(t)=\operatorname{sat}\left(\nu\left(t_{k}\right)\right)\end{array} \quad \forall t \in\left[t_{k}, t_{k+1}\right)\right.$ 
where $\nu(\cdot) \in \mathbb{R}^{m}$ is the output of the controller. In this paper we consider a state-feedback control law:

$\nu\left(t_{k}\right)=K x\left(t_{k}\right)$

where $K \in \mathbb{R}^{m \times n}$ is the gain matrix. The equilibrium point is the origin.

Considering the system formed by (2) and (3), we aim at designing an event-triggered controller, that is, the controller gain and a trigger strategy that defines the trigger instants, to reduce the number of events. In the context of a networked implementation as described in Figure 1, this corresponds to reduce the number of messages exchanged between the nodes, so that we can save communication bandwidth and energy. Thus, we can state the problem we want to address as:

Problem 1 Given a continuous-time stabilizing statefeedback controller for (1), devise an event-triggered implementation of this control law that guarantees the regional (or global, when possible) asymptotic stability of the origin of the closed-loop system (2)-(3) for initial conditions in a given set $\mathcal{X}_{0} \subset \mathbb{R}^{n}$ containing the origin, while reducing the number of data transmission events between the sensor/plant node and the controller node. This is known as emulation design problem.

Problem 2 Devise controller gains and an eventtriggered strategy that guarantee the regional (or global, when possible) asymptotic stability of the origin of the closed-loop system (2)-(3) for initial conditions in a given set $\mathcal{X}_{0} \subset \mathbb{R}^{n}$ containing the origin, while reducing the number of data transmission events between the sensor/plant node and the controller node. This is referred to as co-design problem.

\section{Non-triggered system dynamics}

In this section we recall some stability conditions for the system composed by the direct connection between (1) and a continuous-time implementation of (3), i. e. $\nu(t)=K x(t)$, through a saturating actuator, which leads to the system:

$\dot{x}(t)=A x(t)+B \operatorname{sat}(K x(t))$

Given a gain vector $K,(4)$ is regionally stable in a set $\mathcal{X}_{0}$ if there exist a diagonal matrix $\bar{S}>0 \in \mathbb{R}^{m \times m}$, matrices $\bar{W}=\bar{W}^{\prime}>0 \in \mathbb{R}^{n \times n}, \bar{Z} \in \mathbb{R}^{m \times n}$ satisfying the following relations (see ? for details):

$$
\begin{aligned}
& {\left[\begin{array}{cc}
\operatorname{He}\{(A+B K) \bar{W}\} & B \bar{S}-\bar{Z}^{\prime} \\
\bar{S} B^{\prime}-\bar{Z} & -2 \bar{S}
\end{array}\right]<0} \\
& {\left[\begin{array}{cc}
\bar{W} & \bar{W} K_{(j)}^{\prime}-\bar{Z}_{(j)}^{\prime} \\
* & u_{0(j)}^{2}
\end{array}\right] \geq 0 \quad j=1 \ldots m}
\end{aligned}
$$

and $\mathcal{X}_{0} \subset \mathcal{E}\left(\bar{W}^{-1}\right)$.

In this case, $V(x)=x^{\prime} \bar{W}^{-1} x$ is such that $\dot{V}(x)<$ $0, \forall x \in \mathcal{E}\left(\bar{W}^{-1}\right)$, i. e., $\mathcal{E}\left(\bar{W}^{-1}\right)$ is a contractive invariant set and a region of asymptotic stability (RAS) of the origin for system (4). A RAS of an equilibrium point is a subset of its region of attraction which has a analytical representation and can be used as an estimate for the region of attraction?.

\section{Event-trigger strategy for given controller gains}

In this section, we assume that the gain matrix $K$ has been designed such that (4) is regionally stable in the target set $\mathcal{X}_{0}$. This is guaranteed if $K$ is such that the linear matrix inequalities in (5) are satisfied and $\mathcal{X}_{0} \subset \mathcal{E}\left(\bar{W}^{-1}\right)$. Then we propose an event-triggered strategy and provide stability conditions for the closedloop system under this strategy by means of a quadratic Lyapunov function.

The event trigger strategy consists basically in defining what we call a trigger condition. This condition is therefore continuously monitored. If, at instant $t$, the trigger condition is verified, then the control signal is updated ad we have $t_{k+1}=t$.

Defining $\delta(t)=x\left(t_{k}\right)-x(t)$, i.e., $\delta(t)$ is a measure of the difference between the last sampled state to compute (3) and the current continuous state, in ? the proposed trigger condition consists in verifying if $\|\delta(t)\|=$ $\sigma_{0}\|x(t)\|$. In other words, while $\|\delta(t)\|<\sigma_{0}\|x(t)\|$, i.e., the relative difference between the last sampled state and the current one is lesser than $\sigma_{0}$, the control signal is kept unchanged. In particular, it is shown that this trigger criterion ensures that the closed-loop (2)-(3) is asymptotically stable and that a minimum inter-event time exist (i. e., Zeno solutions are avoided) provided $\sigma_{0}$ is selected from an interval of values determined by the system dynamics.

In this paper, we consider the generalized trigger condition $\delta^{\prime}(t) Q_{\delta} \delta(t)>x^{\prime}(t) Q_{x} x(t)$, where $Q_{\delta}$ and $Q_{x}$ are symmetric positive definite matrices of dimension $n$. In this case, while $\delta^{\prime} Q_{\delta} \delta \leq x^{\prime} Q_{x} x$, the control applied to the plant is kept constant. This generalization adds degrees of freedom and can allow a larger reduction in the sampling activity. The term $\delta^{\prime}(t) Q_{\delta} \delta(t)-x^{\prime}(t) Q_{x} x(t)$ is a relative measure of the deviation between the last sampled state and the current one, with $Q_{\delta}$ and $Q_{x}$ acting as weights. The relation between these matrices plays a role similar to $\sigma_{0}$ in ?: the "larger" $Q_{x}$ and the "smaller" $Q_{\delta}$ the more we let the current state deviate from the last sampled one and the less sampling is expected. Hence, we can 
rewrite our event-trigger strategy in the following more convenient form:

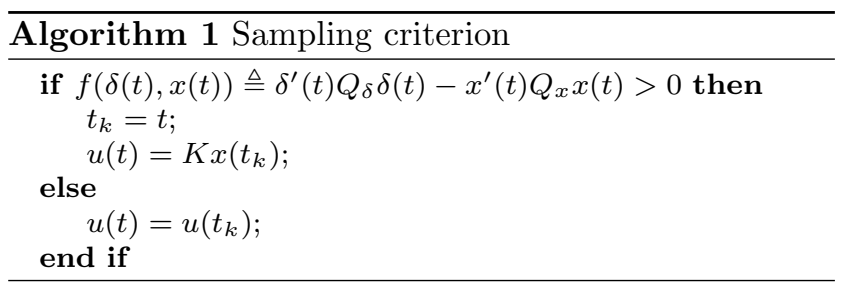

Now we provide conditions that ensure the asymptotic stability of the origin of (2)-(3) when the eventtrigger strategy Algorithm 1 is used.

Theorem 1 If there exist a diagonal positive definite matrix $S \in \mathbb{R}^{m \times m}$, symmetric positive definite matrices $W, \bar{Q}_{x}, Q_{\delta} \in \mathbb{R}^{n \times n}$ and matrices $G_{2}, Z_{1} \in \mathbb{R}^{m \times n}$ such that the following LMIs are satisfied:

$$
\left[\begin{array}{cccc}
\operatorname{He}\{(A+B K) W\} & W & B K & B S-Z_{1}^{\prime} \\
* & -\bar{Q}_{x} & 0 & 0 \\
* & * & -Q_{\delta} & -G_{2}^{\prime} \\
* & * & * & -2 S
\end{array}\right]<0
$$

$$
\left[\begin{array}{cccc}
W & 0 & W K_{(j)}^{\prime}-Z_{1(j)}^{\prime} & W \\
* & Q_{\delta} & K_{(j)}^{\prime}-G_{2(j)}^{\prime} & 0 \\
* & * & u_{0(j)}^{2} & 0 \\
* & * & * & \bar{Q}_{x}
\end{array}\right]>0 \quad j=1 \ldots m
$$

then, $\mathcal{E}\left(W^{-1}\right)=\left\{x \in \mathbb{R}^{n} ; x^{\prime} W^{-1} x \leq 1\right\}$ is a RAS for the system (2)-(3) under the sampling strategy given by Algorithm 1, with $Q_{x}=\bar{Q}_{x}^{-1}$.

Proof Between two trigger events, that is for $t \in$ $\left(t_{k}, t_{k+1}\right)$, we can re-write (2)-(3) as follows:

$$
\begin{aligned}
\dot{x}(t) & =A x(t)+B \operatorname{sat}\left(K x\left(t_{k}\right)\right)= \\
& =A x(t)+B K x\left(t_{k}\right)+B \phi\left(K x\left(t_{k}\right)\right)
\end{aligned}
$$

where $\phi(v) \triangleq \operatorname{sat}(v)-v$ is a dead-zone non-linearity.

Using the relation $\delta(t) \triangleq x\left(t_{k}\right)-x(t)$, we can rewrite (8) as follows:

$$
\dot{x}(t)=(A+B K) x(t)+B K \delta(t)+B \phi\left(K x\left(t_{k}\right)\right)
$$

Considering a quadratic Lyapunov function candidate $V(x(t))=x^{\prime}(t) P x(t)$, and dropping the time dependency arguments for clarity, it follows that:

$$
\begin{aligned}
\dot{V}(x)= & x^{\prime}\left[P(A+B K)+(A+B K)^{\prime} P\right] x+ \\
& +x^{\prime} P B K \delta+x^{\prime} P B \phi\left(K x\left(t_{k}\right)\right)+ \\
& +\delta^{\prime} K^{\prime} B^{\prime} P x+\phi^{\prime}\left(K x\left(t_{k}\right)\right) B^{\prime} P x
\end{aligned}
$$

Notice that the argument of $\phi$ can be written as

$K(x+\delta)=\left[\begin{array}{ll}K & K\end{array}\right]\left[\begin{array}{l}x \\ \delta\end{array}\right] \triangleq K_{a} x_{a}$ where $K_{a} \triangleq\left[\begin{array}{ll}K & K\end{array}\right]$ and $x_{a} \triangleq\left[\begin{array}{l}x \\ \delta\end{array}\right]$.

Hence, provided that $x_{a}$ belongs to the region $\mathcal{S}_{0}=$ $\left\{x_{a} \in \mathbb{R}^{2 n} ;-u_{0} \preceq K_{a} x_{a}-G_{a} x_{a} \preceq u_{0}\right\}$, the following generalized sector condition (??) is verified with respect to the deadzone nonlinearity $\phi$ :

$\phi^{\prime}\left(K_{a} x_{a}\right) T\left(\phi\left(K_{a} x_{a}\right)+G_{a} x_{a}\right) \leq 0$

with $T$ being a diagonal positive definite matrix and $G_{a}=\left[G_{1} G_{2}\right]$ a free matrix of appropriate dimensions.

Assuming $x_{a} \in \mathcal{S}_{0}$ and applying (12), we can write:

$\dot{V}(x) \leq \dot{V}(x)-2 \phi^{\prime}\left(K_{a} x_{a}\right) T\left(\phi\left(K_{a} x_{a}\right)+G_{a} x_{a}\right)$

Thus, from (10) and (13), after some algebraic manipulation, we get:

$$
\begin{aligned}
\dot{V}(x) & \leq x^{\prime}\left[P(A+B K)+(A+B K)^{\prime} P\right] x+ \\
& +x^{\prime} P B K \delta+x^{\prime} P B \phi+ \\
& +\delta^{\prime} K^{\prime} B^{\prime} P x+\phi^{\prime} B^{\prime} P x- \\
& -2 \phi^{\prime} T \phi-2 \phi^{\prime} T G_{1} x- \\
& -2 \phi^{\prime} T G_{2} \delta
\end{aligned}
$$

In matrix form:

$\dot{V}(x) \leq\left[\begin{array}{l}x \\ \delta \\ \phi\end{array}\right]^{\prime} M\left[\begin{array}{l}x \\ \delta \\ \phi\end{array}\right]$

with

$M=\left[\begin{array}{ccc}\operatorname{He}\{P(A+B K)\} & P B K & P B-G_{1}^{\prime} T \\ * & 0 & -G_{2}^{\prime} T \\ * & * & -2 T\end{array}\right]$

From Algorithm 1, for $t \in\left(t_{k}, t_{k+1}\right)$, it follows that $\delta^{\prime} Q_{\delta} \delta-x^{\prime} Q_{x} x \leq 0$. Then, if the following inequality is verified:

$\left[\begin{array}{ccc}\operatorname{He}\{P(A+B K)\}+Q_{x} & P B K & P B-G_{1}^{\prime} T \\ * & -Q_{\delta} & -G_{2}^{\prime} T \\ * & * & -2 T\end{array}\right]<0$

from (15), we can conclude that $\dot{V}(x)<\delta^{\prime} Q_{\delta} \delta-$ $x^{\prime} Q_{x} x \leq 0$ for $t \in\left(t_{k}, t_{k+1}\right)$, provided $x_{a} \in \mathcal{S}_{0}$.

Pre- and post-multiplying (16) by $\operatorname{diag}(W, I, S)$, with $W=P^{-1}, S=T^{-1}$, and considering the variable change $Z_{1}=G_{1} W,(16)$ is equivalent to:

$$
\left[\begin{array}{ccc}
\operatorname{He}\{(A+B K) W\}+W Q_{x} W & B K & B S-Z_{1}^{\prime} \\
* & -Q_{\delta} & -G_{2}^{\prime} \\
* & * & -2 S
\end{array}\right]<0
$$

Applying the Schur's complement to the element $\operatorname{He}\{(A+B K) W\}+W Q_{x} W$ and defining $\bar{Q}_{x} \triangleq Q_{x}^{-1}$, 
we retrieve the matrix inequality $(6)$. Therefore, the satisfaction of (6) implies that $\dot{V}(x)<0$ for $t \in\left(t_{k}, t_{k+1}\right)$, provided that $x_{a}(t) \in \mathcal{S}_{0}$.

At the instants $t=t_{k}$, we have $\delta\left(t_{k}\right)=0$ and the system reduces to (4). Hence, satisfaction of (6) implies that the first relation of $(5)$ holds and that $\dot{V}\left(x\left(t_{k}\right)\right)<$ 0 , provided $x_{a}\left(t_{k}\right) \in \mathcal{S}_{0}$.

Now we show that (7) guarantees that $x_{a}(t) \in$ $\mathcal{S}_{0}, \forall t \geq 0$, provided $x(0) \in \mathcal{E}\left(W^{-1}\right)$.

Pre- and post-multiplying (7) by $\operatorname{diag}(P, I, I, I)$, recalling that $Z_{1}=G_{1} W$ and $\bar{Q}_{x}=Q_{x}^{-1}$, applying Schur's complement twice and recalling that $[K-$ $\left.G_{1} K-G_{2}\right]=K_{a}-G_{a}$, the following relations are verified for $j=1 \ldots m$ :

$$
\left[\begin{array}{cc}
P-Q_{x} & 0 \\
0 & Q_{\delta}
\end{array}\right]-\left(K_{a(j)}-G_{a(j)}\right)^{\prime} \frac{1}{u_{0(j)}^{2}}\left(K_{a(j)}-G_{a(j)}\right)>0
$$

Pre- and post-multiplying (18) by $\left[\begin{array}{ll}x^{\prime} & \delta^{\prime}\end{array}\right]$ and $\left[\begin{array}{l}x \\ \delta\end{array}\right]$ respectively, (18) implies that:

$$
\begin{aligned}
& x^{\prime} P x+\delta^{\prime} Q_{\delta} \delta-x^{\prime} Q_{x} x- \\
& -x_{a}^{\prime}\left(K_{a(j)}-G_{a(j)}\right)^{\prime} \frac{1}{u_{0(j)}^{2}}\left(K_{a(j)}-G_{a(j)}\right) x_{a}>0
\end{aligned}
$$

Hence, if $t \in\left(t_{k}, t_{k+1}\right)$, since $\delta^{\prime} Q_{\delta} \delta-x^{\prime} Q_{x} x \leq 0$, we conclude that $x_{a}(t) \in S_{0}$ if $x(t) \in \mathcal{E}(P)$. On the other hand, at $t=t_{k}$, we have $\delta(t)=0$ and it also follows that $x_{a}\left(t_{k}\right) \in \mathcal{S}_{0}$ if $x\left(t_{k}\right) \in \mathcal{E}(P)$. Thus, we can conclude that if $x(0) \in \mathcal{E}(P)$, (6) along with (7) effectively ensures that $x_{a}(t) \in \mathcal{S}_{0}$ and $\dot{V}(x)<0, \forall t \geq 0$, which ensures that $\mathcal{E}(P)$ is an invariant and contractive set with respect to the system (2)-(3), being included in its region of attraction.

Theorem 1 ensures the regional asymptotic stability of the closed-loop system and can be applied to both stable or unstable plants. Nevertheless, if $A_{p}$ is Hurwitz, global stability can be achievable ?. A sufficient condition for the global stability of the closed-loop system under the event-trigger strategy can therefore be stated as a corollary to Theorem 1 as follows.

Corollary 1 If there exist a diagonal positive definite matrix $S \in \mathbb{R}^{m \times m}$, symmetric positive definite matrices $W, \bar{Q}_{x}, Q_{\delta} \in \mathbb{R}^{n \times n}$ such that the following $L M I$ is satisfied:

$$
\left[\begin{array}{cccc}
\operatorname{He}\{(A+B K) W\} & W & B K & B S-W K^{\prime} \\
* & -\bar{Q}_{x} & 0 & 0 \\
* & * & -Q_{\delta} & -K^{\prime} \\
* & * & * & -2 S
\end{array}\right]<0
$$

then, the origin of system (2)-(3) is globally asymptotically stable under the sampling strategy given by Algorithm 1, with $Q_{x}=\bar{Q}_{x}^{-1}$.

Proof It follows the same lines as the proof of Theorem 1 but applying a global generalized sector condition, i. e., using $G_{a}=K_{a}$, which implies $G_{1}=G_{2}=K$. In this case, (12) is valid $\forall x_{a} \in \mathbb{R}^{2 n}$.

Remark 1 The existence of a lower bound for the interevent times can be proved applying the same arguments used in ? and taking into account that the event-trigger criterion satisfies:

$\|\delta(t)\|^{2} \leq \frac{\lambda_{\max }\left(Q_{x}\right)}{\lambda_{\min }\left(Q_{\delta}\right)}\|x(t)\|^{2}$

Hence, the possibility of Zeno solutions is eliminated in the proposed strategy.

\subsection{Optimization problems}

Considering a region of admissible initial states $\mathcal{X}_{0}$, if $\mathcal{X}_{0} \subset \mathcal{E}\left(W^{-1}\right)$ and conditions of Theorem 1 are satisfied, $\mathcal{X}_{0}$ is also included in the region of attraction of the origin of the closed-loop system. Moreover, to address Problem 1, we also aim at reducing the number of transmission events.

As presented at the beginning of Section 4, the reduction of the transmission activity can be obtained by aiming to find $Q_{x}$ as "large" as possible and $Q_{\delta}$ as "small" as possible, while ensuring the stability of the origin of the closed-loop system under the eventtrigger strategy. Considering $\mathcal{X}_{0}$ specified as an ellipsoid $\mathcal{E}\left(P_{0}\right)=\left\{x \in \mathbb{R}^{n} ; \quad x^{\prime} P_{0} x \leq 1\right\}$, with $P_{0}=P_{0}^{\prime}>0$, and restricting $Q_{x}=\alpha^{-1} I$ and $Q_{\delta}=\mu I$, with $\alpha, \mu \in \mathbb{R}$, this goal can be achieved from the following convex optimization problem:

$$
\min _{\left\{W, \alpha, \mu, S, G_{2}, Z_{1}\right\}}(\alpha+\mu)
$$

subject to:

$$
\text { (6), (7), } W-P_{0}^{-1}>0
$$

Notice that this optimization problem cannot be normalized to eliminate one of the variables, $\alpha$ or $\mu$, due to the terms $B K$ and $K^{\prime} B^{\prime}$ which do not involve any variable. The last constraint in (22) ensures $\mathcal{X}_{0} \subset \mathcal{E}\left(W^{-1}\right)$.

Furthermore, we can add degrees of freedom by letting both $Q_{x}$ and $Q_{\delta}$ be generic positive definite matrices. This leads to the following optimization problem:

$$
\min _{\left\{W, \bar{Q}_{x}, Q_{\delta}, S, G_{2}, Z_{1}\right\}} \operatorname{tr}\left(\bar{Q}_{x}+Q_{\delta}\right)
$$

subject to:

$$
\text { (6), (7), W- } P_{0}^{-1}>0
$$


In this case, the minimization of the trace of $\bar{Q}_{x}=$ $Q_{x}^{-1}$ implies the maximization of the trace of $Q_{x}$, which is the primary objective.

\section{Co-design}

If the controller gain $K$ is a variable, conditions established by Theorem 1 are not LMIs, hence the optimization problems proposed in Section 4.1 cannot be used to obtain the controller and trigger parameters simultaneously, as they become non-convex. Nevertheless, some modifications can be made to linearize the conditions, leading to the following result:

Theorem 2 If there exist a diagonal positive definite matrix $S \in \mathbb{R}^{m \times m}$, symmetric positive definite matrices $W, \bar{Q}_{x}, \bar{Q}_{\delta} \in \mathbb{R}^{n \times n}$ and matrices $Y, Z_{1}, Z_{2} \in \mathbb{R}^{m \times n}$ such that the following LMIs are satisfied:

$$
\begin{aligned}
& {\left[\begin{array}{cccc}
\operatorname{He}\{A W+B Y\} & W & B Y & B S-Z_{1}^{\prime} \\
* & -\bar{Q}_{x} & 0 & 0 \\
* & * & -\bar{Q}_{\delta} & -Z_{2}^{\prime} \\
* & * & * & -2 S
\end{array}\right]<0} \\
& {\left[\begin{array}{cccc}
W & 0 & Y_{(j)}^{\prime}-Z_{1(j)}^{\prime} & W \\
* & \bar{Q}_{\delta} & Y_{(j)}^{\prime}-Z_{2(j)}^{\prime} & 0 \\
* & * & u_{0(j)}^{2} & 0 \\
* & * & * & \bar{Q}_{x}
\end{array}\right]>0 \quad j=1 \ldots m}
\end{aligned}
$$

then, $\mathcal{E}\left(W^{-1}\right)=\left\{x \in \mathbb{R}^{n} ; x^{\prime} W^{-1} x \leq 1\right\}$ is a $R A S$ for the system (2)-(3) under the sampling strategy given by Algorithm 1, with $Q_{x}=\bar{Q}_{x}^{-1}, Q_{\delta}=W^{-1} \bar{Q}_{\delta} W^{-1}$ and $K=Y W^{-1}$.

Proof Pre- and post-multiplying (6) by $\operatorname{diag}(I, I, W, I)$, recalling $Z_{1}=G_{1} W$ and $\bar{Q}_{x}=Q_{x}^{-1}$ and making the variable substitutions $\bar{Q}_{\delta}=W Q_{\delta} W, Y=K W$ and $Z_{2}=G_{2} W$ one obtains that (6) and (24) are equivalent. Pre- and post-multiplying ( 7$)$ by $\operatorname{diag}(I, W, I, I)$, and making the same variable substitutions one concludes that (7) and (25) are equivalent.

\subsection{Optimization problems for co-design}

Theorem 2 cannot be cast directly into an optimization problem like those of Section 4.1 because $Q_{\delta}$ does not appear as a decision variable and $\bar{Q}_{\delta}=W Q_{\delta} W$ is not affine on $W$ and $Q_{\delta}$, meaning that the optimization objectives would become non-convex. Nevertheless, we can optimize $Q_{\delta}$ indirectly by minimizing the trace of
$\bar{Q}_{\delta}$. Thus, we propose the following optimization problem:

$$
\min _{\left\{W, \bar{Q}_{x}, \bar{Q}_{\delta}, Y, Z, Z_{2}, S\right\}} \operatorname{tr}\left(\bar{Q}_{x}+\bar{Q}_{\delta}\right)
$$

subject to:

$$
\text { (24), (25), } W-P_{0}^{-1}>0
$$

Remark 2 When $A_{p}$ is Hurwitz, the same procedures used so far can be applied to develop a similar optimization problem guaranteeing global stability. In this case, additional conditions, e. g. convergence rate conditions, must be included in the optimization problem to turn it well-posed.

Remark 3 The present results (for $K$ given and the codesign) can be straightforwardly extended to the case of an uncertain system described by a polytopic model, i.e. if

$A \in \operatorname{Co}\left\{A_{1}, A_{2}, \ldots, A_{N}\right\}$

$B \in \operatorname{Co}\left\{B_{1}, B_{2}, \ldots, B_{M}\right\}$

and the conditions stated in Theorem 1, in Corolary 1 or in Theorem 2 are verified at the vertices of polytopes (27) and (28), that is, $\forall\left(A_{i}, B_{j}\right)$, with $i=$ $1, \ldots, N$ and $j=1, \ldots, M$, by convexity arguments, one can conclude that they are verified for the entire polytope.

\section{Numerical example}

We illustrate the application of the proposed results considering the following continuous-time unstable plant and $u_{0}=5$ :

$\dot{x}(t)=\left[\begin{array}{ll}0 & 1 \\ 4 & 0\end{array}\right] x+\left[\begin{array}{l}1 \\ 0\end{array}\right] \operatorname{sat}(u(t))$

We choose $K=[-9-5.500]$ so that the plant without saturation and with a continuous-time controller is stabilized with closed-loop poles in -3 and -6 . We consider $\mathcal{X}_{0}$ as the disk around the origin with radius 1 , that is $\mathcal{X}_{0}=\left\{x \in \mathbb{R}^{n} ; \quad x^{\prime} x \leq 1\right\}$. From this data, the optimization problem (22) gives:

$$
\begin{gathered}
Q_{x}=\left[\begin{array}{cc}
0.3256 & 0 \\
0 & 0.3256
\end{array}\right] \quad Q_{\delta}=\left[\begin{array}{cc}
5.138 & 0 \\
0 & 5.138
\end{array}\right] \\
W^{-1}=\left[\begin{array}{ll}
0.6490 & 0.2472 \\
0.2472 & 0.5146
\end{array}\right]
\end{gathered}
$$

Figure 2 shows results of simulation for $x(0)=$ $[\sqrt{2} / 2 \sqrt{2} / 2]^{\prime}$ (notice that $x(0) \in \mathcal{X}_{0}$ ). Only 26 events occurred in the time interval $[0,2]$. For comparison purposes, the response considering a non-triggered (i.e., 


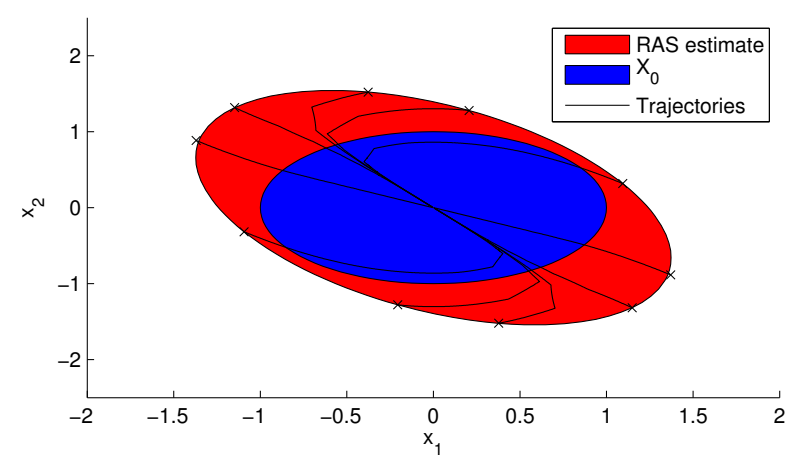

Fig. 3 RAS with event-trigger tunned using (22)

continuous) controller implementation is also depicted in this figure. We can observe that no significant degradation is introduced by the event-triggered implementation. Figure 3 shows the set $\mathcal{E}\left(W^{-1}\right)$ obtained. Note that $\mathcal{E}\left(W^{-1}\right)$ contains $\mathcal{X}_{0}$, as expected, and that it does not touch $\mathcal{X}_{0}$, which might mean that there is some conservatism induced by the trigger parameters obtained with (22).

We now use optimization problem (23) to tune the trigger, obtaining:

$$
\begin{gathered}
Q_{x}=\left[\begin{array}{cc}
0.6109 & -0.0631 \\
-0.0631 & 0.4065
\end{array}\right] \quad Q_{\delta}=\left[\begin{array}{ll}
4.519 & 2.761 \\
2.761 & 1.688
\end{array}\right] \\
W^{-1}=\left[\begin{array}{ll}
0.8464 & 0.2080 \\
0.2080 & 0.7184
\end{array}\right]
\end{gathered}
$$

With these values, we obtain a less conservative solution in terms of the triggering activity, as shown in figures 4 and 5 . Now only 9 events occurred in the time interval $[0,2]$. On the other hand, as expected, the RAS obtained is much closer to $\mathcal{X}_{0}$ than with parameters tunned using (22), since typically there is a trade off between the number of events and the size of the region of attraction. One also observes a slightly larger degradation relative to the continuous-time implementation than that obtained with the previous optimization problem.

Table 1 shows a comparison of the number of events and the minimum inter-event times obtained with each of the optimization problems for some initial conditions. It gives evidence that the optimization problem (23) leads to better results in terms of the reduction of the number of events. One can also see that this optimization problem also leaded to a larger minimum interevent time (36 ms, against $25 \mathrm{~ms}$ from the other). Note that this table includes initial conditions that are not in $\mathcal{X}_{0}$ but are in the region of attraction of the closed-loop system.
Table 1 Comparison of optimization problems

\begin{tabular}{ccccc}
\hline$x(0)$ & $\begin{array}{l}\text { Number } \\
\text { of } \\
\text { events } \\
\text { OP }(22)\end{array}$ & $\begin{array}{l}\text { Minimum } \\
\text { inter-event } \\
\text { time (ms) } \\
\text { OP }(22)\end{array}$ & $\begin{array}{l}\text { Number } \\
\text { of } \\
\text { events } \\
\text { OP }(23)\end{array}$ & $\begin{array}{l}\text { Minimum } \\
\text { inter-event } \\
\text { time (ms) } \\
\text { OP }(23)\end{array}$ \\
\hline$(-3.00,3.00)$ & 35 & 35 & 12 & 71 \\
$(-2.00,1.00)$ & 31 & 30 & 14 & 41 \\
$(-2.00,2.00)$ & 28 & 51 & 6 & 146 \\
$(-1.00,0.00)$ & 30 & 25 & 15 & 36 \\
$(-1.00,1.00)$ & 28 & 51 & 11 & 85 \\
$(-1.00,2.00)$ & 25 & 79 & 8 & 136 \\
$(0.00,-1.00)$ & 25 & 63 & 11 & 67 \\
$(0.00,1.00)$ & 25 & 63 & 11 & 67 \\
$(1.00,-2.00)$ & 25 & 79 & 8 & 136 \\
$(1.00,-1.00)$ & 28 & 51 & 11 & 85 \\
$(1.00,0.00)$ & 30 & 25 & 15 & 36 \\
$(2.00,-2.00)$ & 28 & 51 & 6 & 146 \\
$(2.00,-1.00)$ & 31 & 30 & 14 & 41 \\
$(3.00,-3.00)$ & 35 & 35 & 12 & 71 \\
\hline
\end{tabular}

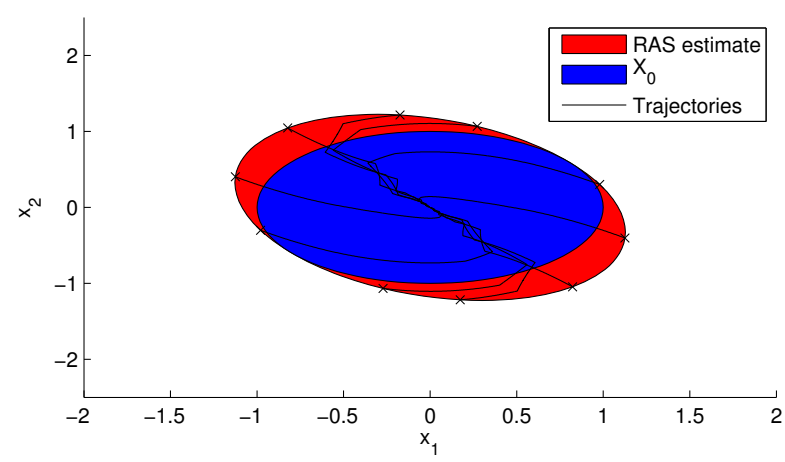

Fig. 5 RAS with event-trigger tunned using (23)

Finally, using the co-design optimization problem (26), we obtain:

$$
\begin{aligned}
& Q_{x}=\left[\begin{array}{cc}
0.6463 & -0.05563 \\
-0.05563 & 0.3943
\end{array}\right] \quad Q_{\delta}=\left[\begin{array}{ll}
3.371 & 2.444 \\
2.444 & 1.773
\end{array}\right] \\
& W^{-1}=\left[\begin{array}{ll}
0.8812 & 0.1983 \\
0.1983 & 0.6689
\end{array}\right] \quad K=[-5.483-3.976]
\end{aligned}
$$

Figure 6 shows the results of simulation using these parameter values and the same initial condition $x(0)=$ $[\sqrt{2} / 2 \sqrt{2} / 2]^{\prime}$. In this case, 14 events occurred in the interval $[0,2]$ and the response deviates a little more from the continuous-time implementation than the previous results. Figure 7 shows the set $\mathcal{E}\left(W^{-1}\right)$ obtained with the co-design optimization problem. Table 2 shows the number of events and the minimum inter-event times obtained from simulations with various initial conditions. Comparing to the results obtained from optimization problem (26), listed in Table 1, one can see that the co-design leaded to worse numbers of events (an average of 14.2 events against 11 when optimization problem (26) is used), but to better minimum inter-event times (68 ms against $36 \mathrm{~ms}$ ). Note that Table 2, as Table 1, 

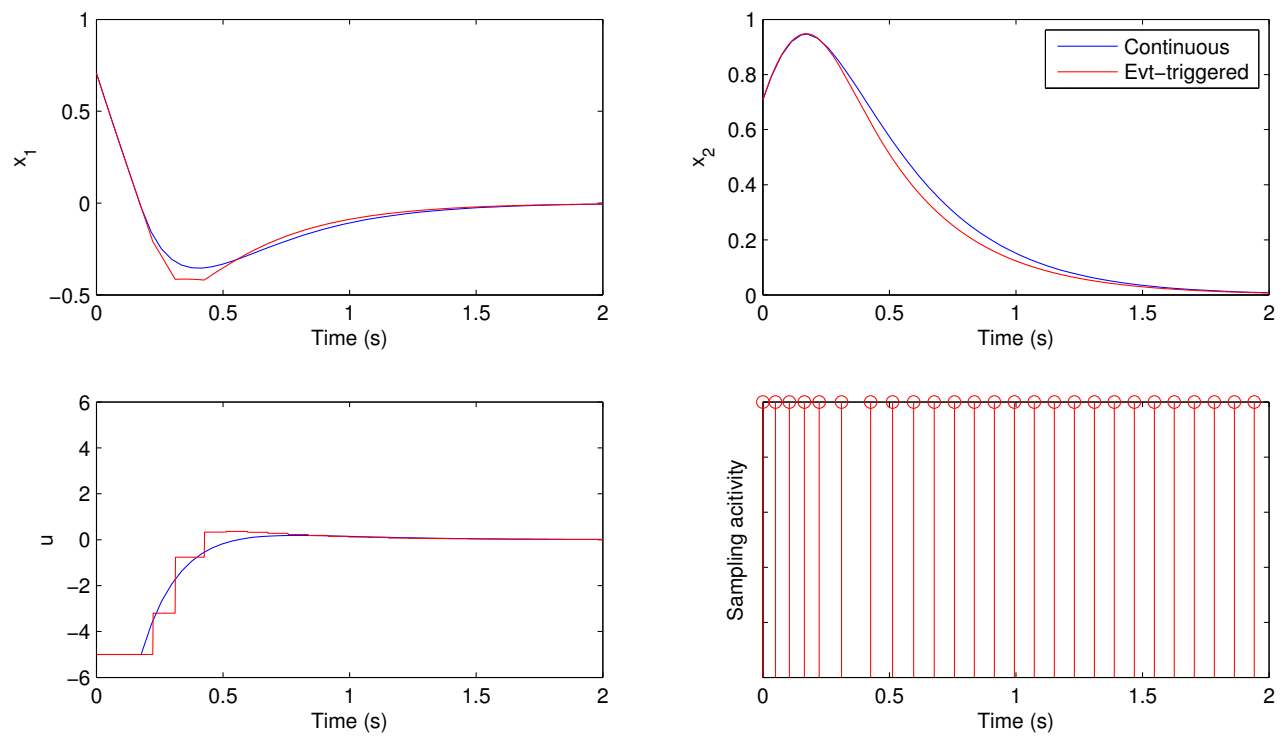

Fig. 2 Simulation results - Comparison between continuous system and event-trigger tunned via (22)
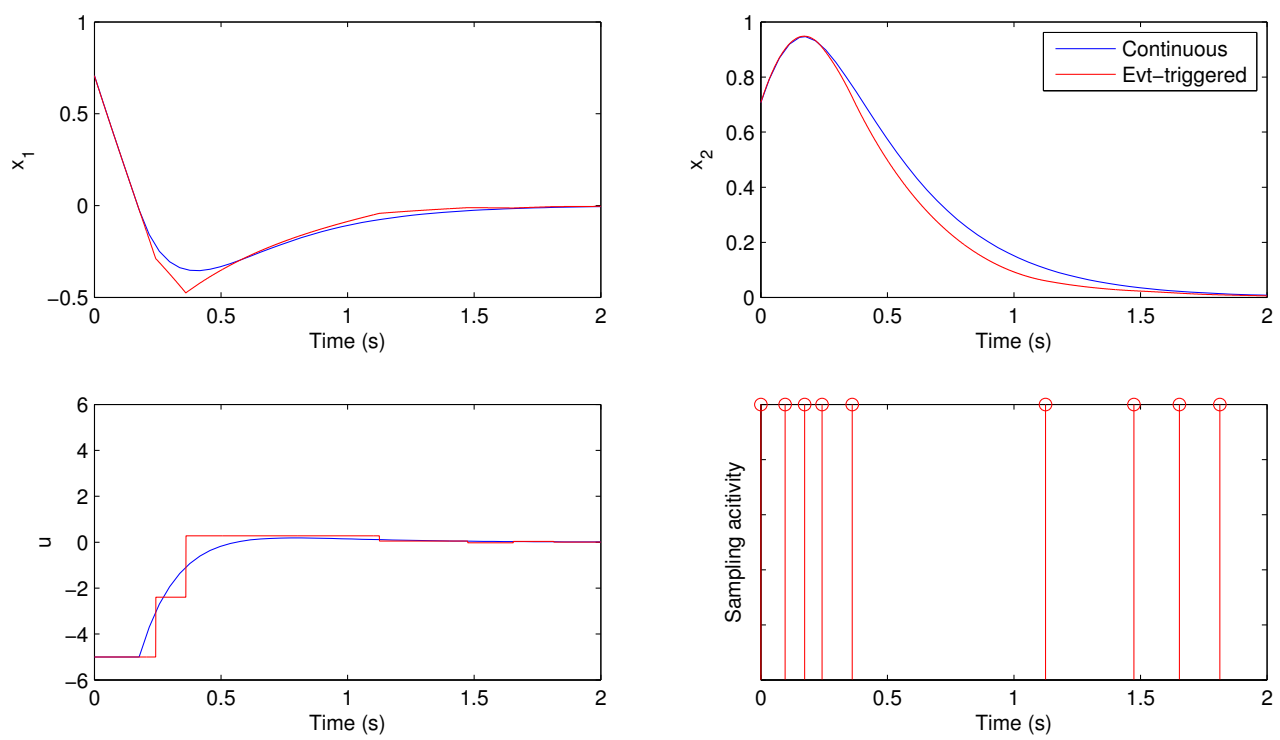

Fig. 4 Simulation results - Comparison between continuous system and event-trigger tunned via (23)

includes initial conditions that are not in $\mathcal{X}_{0}$ but are in the region of attraction of the closed-loop system.

\section{Concluding remarks}

In this paper we proposed a methodology to design event-trigger strategies for state-feedback controlled plants subject to input saturation. The method guarantees regional asymptotic stability of the origin for a given set of initial conditions when the plant is unstable. For stable plants, we show how the method can be extended to achieve global stability.
Since the stability conditions are presented in the form of LMIs, convex optimization problems can be proposed to compute the trigger function parameters aiming at a reduction of the number of events (and, therefore, of the data transmissions in a networked implementation) while ensuring the stability of the origin of the closed-loop system. Two different optimization problems are proposed for the case in which the controller gain is defined a priori. Numerical experiments show that the results obtained with the more generic one can lead to a reduction of the number of events.

Stability conditions that allow the simultaneous design of the controller gains and the trigger parameters are also presented. In this case, part of the trig- 

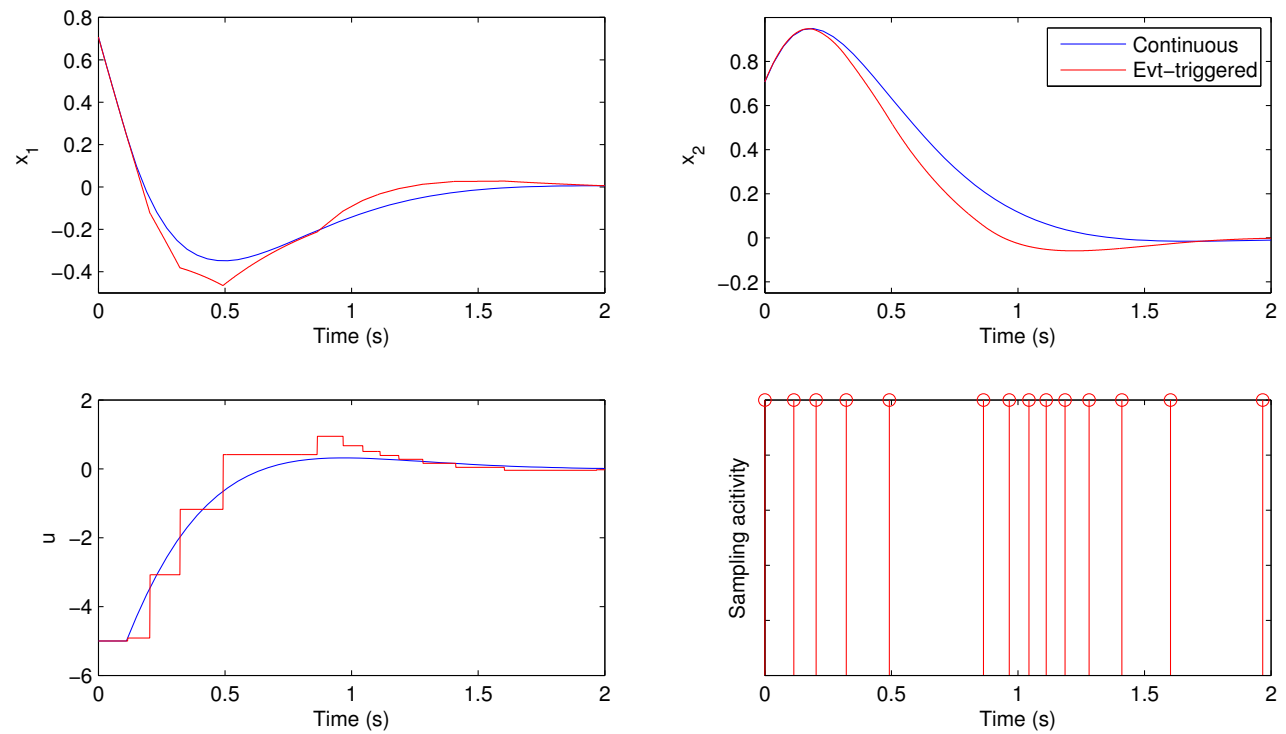

Fig. 6 Simulation results - Comparison between continuous system and event-trigger tunned via (26) - co-design

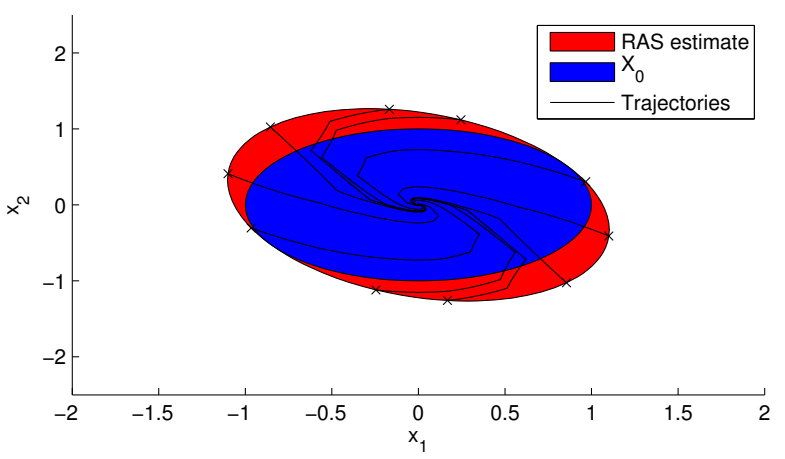

Fig. 7 RAS with event-trigger tunned using (26) - co-design

ger parameters do not appear explicitly in the stability conditions, but since a monotonic function of them do appear, we still can propose a suitable convex optimization problem based on LMI restrictions to tune the controller and the trigger. Numerical experiments show that the co-design method proposed does not always lead to an effective reduction of the number of samples when compared to the case where the controller gain is given; nevertheless, the experiments show that the co-design indeed leads to an effective increase of the minimum inter-event time.

We have also shown that the results can be straightforwardly extended to uncertain systems described by polytopic models. This is possible due to the linear nature of the constraints in the stability theorems.

Future work will address the extension of the proposed approaches to systems with delays to model the communication network and the data computation times.
Table 2 Number of events and inter-event times - co-design

\begin{tabular}{ccc}
\hline$x(0)$ & $\begin{array}{c}\text { Number of } \\
\text { events for } \\
t \in[0,2]\end{array}$ & $\begin{array}{c}\text { Minimum } \\
\text { inter-event time } \\
(\mathrm{ms})\end{array}$ \\
\hline$(-3.00,4.00)$ & 15 & 68 \\
$(-3.00,3.00)$ & 13 & 68 \\
$(-3.00,5.00)$ & 16 & 69 \\
$(-2.00,2.00)$ & 13 & 68 \\
$(-2.00,3.00)$ & 16 & 68 \\
$(-2.00,5.00)$ & 15 & 69 \\
$(-1.00,0.00)$ & 14 & 68 \\
$(-1.00,1.00)$ & 13 & 68 \\
$(-1.00,2.00)$ & 12 & 68 \\
$(-1.00,3.00)$ & 14 & 68 \\
$(0.00,-2.00)$ & 14 & 68 \\
$(0.00,-1.00)$ & 15 & 69 \\
$(0.00,1.00)$ & 15 & 69 \\
$(0.00,2.00)$ & 14 & 68 \\
$(1.00,-3.00)$ & 14 & 68 \\
$(1.00,-2.00)$ & 12 & 68 \\
$(1.00,-1.00)$ & 13 & 68 \\
$(1.00,0.00)$ & 14 & 69 \\
$(2.00,-5.00)$ & 15 & 68 \\
$(2.00,-3.00)$ & 16 & 68 \\
$(2.00,-2.00)$ & 13 & 69 \\
$(3.00,-5.00)$ & 16 & 68 \\
$(3.00,-4.00)$ & 15 & 68 \\
$(3.00,-3.00)$ & 13 & \\
\hline
\end{tabular}

